\title{
Scanning stereo-PLIF method for free surface measurements in large 3D domains
}

\author{
Mike van Meerkerk $^{1} \cdot$ C. Poelma ${ }^{1}\left[\right.$ D. J. Westerweel ${ }^{1}$
}

Received: 17 June 2019 / Revised: 18 September 2019 / Accepted: 1 November 2019 / Published online: 6 January 2020

(c) The Author(s) 2019

\begin{abstract}
In this work, we extend a planar laser-induced fluorescence method for free surface measurements to a three-dimensional domain using a stereo-camera system, a scanning light sheet, and a modified self-calibration procedure. The stereo-camera set-up enables a versatile measurement domain with self-calibration, improved accuracy, and redundancy (e.g., possibility to overcome occlusions). Fluid properties are not significantly altered by the fluorescent dye, which results in a non-intrusive measurement technique. The technique is validated by determining the free surface of a hydraulic flow over an obstacle and circular waves generated after droplet impact. Free surface waves can be accurately determined over a height of $L=100 \mathrm{~mm}$ in a large two-dimensional domain $\left(y(x, z)=120 \times 62 \mathrm{~mm}^{2}\right)$, with sufficient accuracy to determine small amplitude variations $(\eta \approx 0.2 \mathrm{~mm})$. The temporal resolution $(\Delta t=19 \mathrm{~ms})$ is only limited by the available scanning equipment $(f=1 \mathrm{kHz}$ rate). For other applications, this domain can be scaled as needed.
\end{abstract}

\section{Graphic abstract}

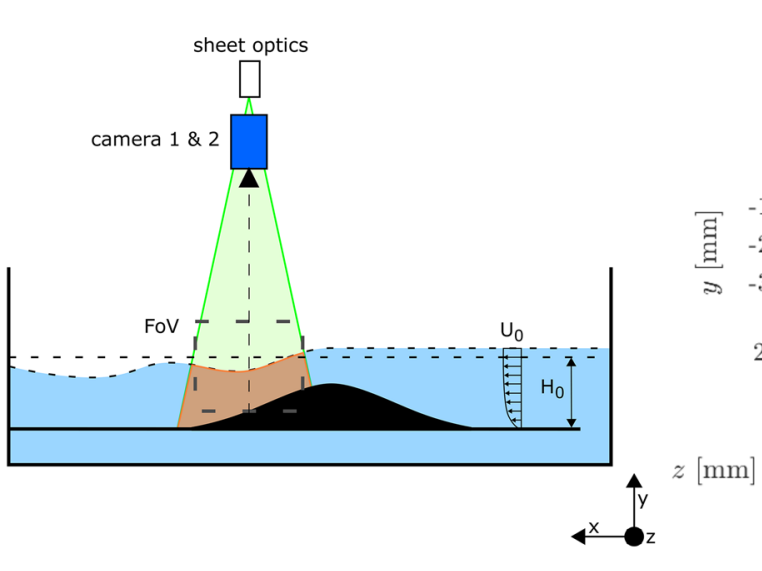

Electronic supplementary material The online version of this article (https://doi.org/10.1007/s00348-019-2846-7) contains supplementary material, which is available to authorized users.

C. Poelma

c.poelma@tudelft.nl

Mike van Meerkerk

M.vanMeerkerk@tudelft.nl

1 Delft University of Technology, 3ME-PE, Leeghwaterstraat 21, 2628CA Delft, The Netherlands

\section{Introduction}

Small-scale free surface dynamics play a significant role in many applications. For example, they strongly influence the response of structures to wave impacts (Lafeber et al. 2012), and the transfer of heat, momentum, mass, and energy between the ocean and atmosphere (Buckley and Veron 2016; Jähne and Haußecker 1998). Therefore, measurements of the small-scale free surface dynamics are required. These measurements are typically performed with intrusive point measurements (e.g., resistive wave probes). 
Fig. 1 General measurement principle of free surface measurement techniques. a Refraction-based techniques relate the slope change to a change in height. b Stereo-correlationbased techniques determine the three-dimensional world position of particles or naturally present features. c Projectionbased techniques determine the free surface height with a projected pattern

Table 1 Overview of selected free surface measurement techniques

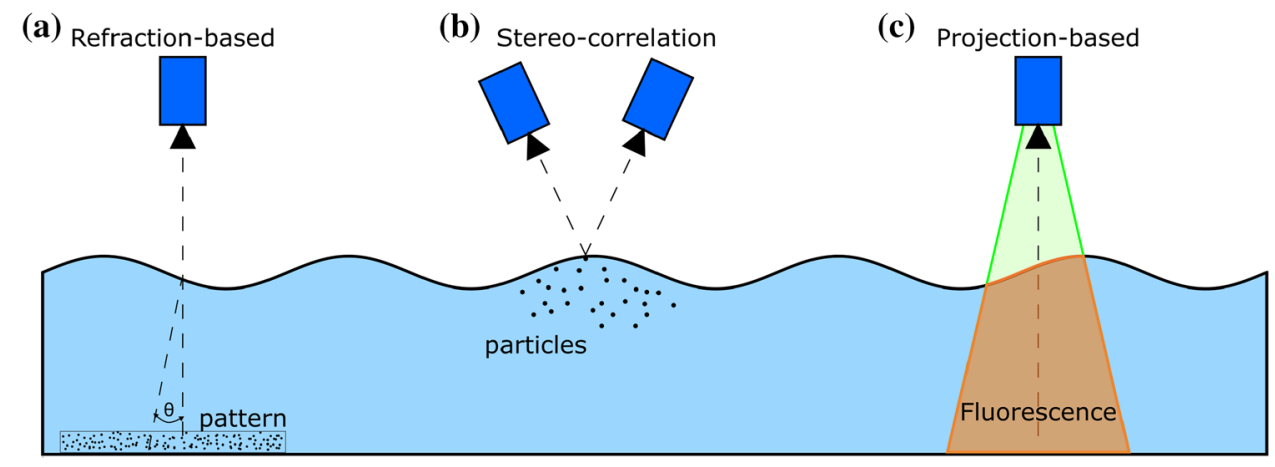

\begin{tabular}{lllll}
\hline Method & $\begin{array}{l}\text { Domain size }(x \times y) \\
\mathrm{m}^{2}\end{array}$ & $\begin{array}{l}\text { Frequency }(f) \\
\mathrm{Hz}\end{array}$ & $\begin{array}{l}\text { Amplitude }(A) \\
\mathrm{m} \times 10^{-3}\end{array}$ & $\begin{array}{l}\text { Accuracy }(\Delta A / A) \\
\%\end{array}$ \\
\hline $\begin{array}{l}\text { Refraction-based techniques } \\
\text { Laser beam refraction }\end{array}$ & & & \\
Savelsberg et al. (2006) & 0.05 & 100 & $O(1)$ & - \\
Free surface synthetic Schlieren & & & \\
$\quad$ Moisy et al. (2009) & $0.2 \times 0.2$ & 100 & $O(0.01-1)$ & $O(2)$ \\
Gomit et al. (2013) & $0.2 \times 0.2$ & 10 & $O(13)$ & $O(3)^{*}$ \\
& $0.4 \times 0.5$ & 10 & $O(10)^{*}$ & $O(7)^{*}$ \\
Stereo-correlation techniques & & & & \\
Benetazzo et al. (2012) & $35 \times 35$ & $5-10$ & $O\left(10^{3}\right)$ & $O(1-2)$ \\
Turney et al. (2009) & $0.07 \times 0.7$ & 250 & $O(10)$ & $O(10)$ \\
Douxchamps et al. (2005) & $0.5 \times 0.5$ & 25 and 250 & $O(40)$ & $O(10-15)$ \\
Zavadsky et al. (2017) & $0.25 \times 0.4$ & 100 & $O(10)$ & $O(15-30)^{*}$ \\
Projection-based techniques & & & & \\
Gomit et al. (2015) & $4 \times 8$ & $O(0.01)$ & $O(130)$ & $O(8)$ \\
Dabiri and Gharib (2001) & $0.18 \times 0.18$ & 30 & 1.2 & $O(6)$ \\
Cobelli et al. (2009) & $0.3 \times 0.45$ & $4 \times 10^{3}$ & $O(100)$ & $O(0.2)$ \\
Tsubaki and Fujita (2005) & $O(0.14 \times 0.18)$ & 12 & $O(10)$ & $O(10)$ \\
André and Bardet (2014) & 0.0075 & $8 \times 10^{3}$ & $O(1)^{*}$ & $O(1)^{*}$ \\
Buckley and Veron (2017) & 0.51 & 7.2 & $O(1-20)$ & $O(1-10)^{*}$ \\
Duncan et al. (1999) & 0.12 & 500 & $O(50)^{*}$ & $O(1)^{*}$ \\
\hline
\end{tabular}

Several parameters are estimated from data presented in each paper, which is denoted by an asterisk (*)

A parameter that was not defined and could not be estimated is denoted by (-)
However, instantaneous wave height measurements can be obtained with optical techniques that are non-intrusive, and therefore preferential in many applications. These optical techniques can be categorized as refraction, stereo-correlation or projection based (Fig. 1).

Techniques based on refraction relate the slope change of a liquid free surface to the refraction of light. A one-dimensional collimated light source (e.g., laser beam) is typically used to determine the deflection on position-sensing photodiodes (Liu et al. 1993) or a camera. A collection of points can be obtained by rapidly scanning the light source over a line (Savelsberg et al. 2006). The technique can be extended to a two-dimensional domain by imaging a pattern through a refractive medium. The patterns are either created by a random-dot pattern (Moisy et al. 2009) or a distribution of particles in a flow (Gomit et al. 2013). Advantages of refraction-based techniques are the ease-of-implementation, the relatively large domain size, and the small error (Table 1). However, ray crossing, which occurs when either the surface curvature or the surface-to-pattern distance becomes too large, limits the measurement of steep and large amplitude waves (Moisy et al. 2009).

Stereo-correlation techniques either use stereo-vision to triangulate characteristic features, such as ocean whitecaps (Benetazzo et al. 2012), or use particle image velocimetry (PIV)/particle tracking velocimetry (PTV) approaches to 
determine the free surface height. The particles used in these approaches are often buoyant (Turney et al. 2009) or neutrally buoyant (Douxchamps et al. 2005). The effect of particles on surface tension can be minimized by ensuring that the particles are hydrophobic. However, particle clustering can occur in regions of convergence of interfacial velocity, which can result in local variance of fluid properties (Turney et al. 2009). The stereo-correlation techniques can obtain free surface heights over large spatial domains (Table 1). However, considerable effort is required to obtain appropriate illumination. Furthermore, the sensitivity to small amplitude free surface disturbances is limited (Zavadsky et al. 2017).

Lastly, projection-based techniques project an image or pattern on a liquid free surface. No surface seeding is required when there are naturally present contaminants (Gomit et al. 2015) or when the reflection of colored light is used (Dabiri and Gharib 2001). Otherwise, a dye is required to make the liquid fully opaque (Cobelli et al. 2009; Tsubaki and Fujita 2005) or to make the liquid fluorescent (André and Bardet 2014; Buckley and Veron 2017; Duncan et al. 1999). For fully opaque fluids, fringe projection techniques can be applied to obtain accurate, two-dimensional free surface height measurements in large three-dimensional domains (Cobelli et al. 2009). For fluorescent fluids, accurate line measurements can easily be obtained with planar laser-induced fluorescence (PLIF) techniques (Duncan et al. 1999). Furthermore, the fluorescent dye does not change the fluid properties as the concentration of dye is typically low.

So far, PLIF-based free surface measurement techniques have been limited to line measurements. The technique offers accurate free surface height measurements, without altering the fluid properties or the need for fixed patterns. Furthermore, per-pixel wave height measurements can be obtained along a line (Buckley and Veron 2017). In contrast, stereo-correlation-based approaches use particles or features and are limited by the distribution of these particles or features over the surface. The accuracy of PLIF-based techniques can even be improved with detection methods relying on light sheet properties (e.g., André and Bardet 2014). However, the PLIF-based approaches are currently limited to planar domains.

This study presents a two-dimensional PLIF-based approach, which applies a stereo-camera set-up with a scanned light sheet (Brücker 1996). Conventional free surface measurements approaches are summarized in Table 1. The current technique can be used in experiments with limited optical access, due to the small camera separation angle. A multi-step calibration procedure is used to reduce the calibration requirements (Hori and Sakakibara 2004; Wieneke 2008). These optical limitations are imposed by future work in the newly build Multiphase Wave Lab at MARIN in The Netherlands. The technique is also evaluated for typical optical conditions.
Measurements can be obtained in relatively large three-dimensional domains $((x, y, z)=(120,100,62) \mathrm{mm})$ with sufficient accuracy to resolve small amplitude waves $(\eta \approx 0.2 \mathrm{~mm})$.

The paper is organized as follows. The experimental setup and equipment are introduced in Sect. 2. This section also describes the calibration procedure. Section 3 shows and discusses the measurements of a hydraulic flow over an obstacle and the waves generated by a droplet impact are shown. Finally, the last section presents some concluding remarks.

\section{Method}

The method allows one to extend one-dimensional height measurements $(y(x, t))$ typically obtained with PLIF (Table 1) to a two-dimensional domain $(y(x, z, t))$. In essence, this could be achieved with a single camera and a multi-plane calibration procedure. However, a stereo-camera set-up allows one to apply a broad calibration that can be refined with an adapted self-calibration procedure as the $z$-direction is constrained by the scanned light sheet. Furthermore, the accuracy of the method can be improved with a stereo-camera set-up. This results in a robust method that can, with some adjustments, be used in applications with occlusions.

The experimental set-up and equipment are introduced in this section. Furthermore, the method to obtain stereoPLIF measurements is detailed. First, the relevant equipment is introduced. Then, the edge detection procedure is introduced, which is required for the adapted self-calibration procedure and measurements. Finally, the stereo-PLIF calibration is detailed, which uses an adapted self-calibration procedure.

\subsection{Flow facility}

Figure 2 shows the experimental set-up used in this study. The experiments are performed in the water tunnel of the Laboratory for Aero- and Hydrodynamics at the Delft University of Technology. The test section has an area of $0.6 \times 0.6 \mathrm{~m}^{2}$ and a length of $5 \mathrm{~m}$. A false bottom, $190 \mathrm{~mm}$ above the channel bottom, is used to generate a defined boundary layer, and to allow the water tunnel to be operated at a reduced water depth.

Free surface waves are generated behind an obstacle, which is mounted on the false bottom at a distance of $0.85 \mathrm{~m}$ from the leading edge. The shape of the obstacle is defined by a fourth-order polynomial

$y(x)=H_{b}\left(1-2(x / L)^{2}+(x / L)^{4}\right)$,

with $H_{b}=0.117 \mathrm{~m}$ the obstacle height, and $L=0.295 \mathrm{~m}$ the obstacle half-length (Gui et al. 2014). 


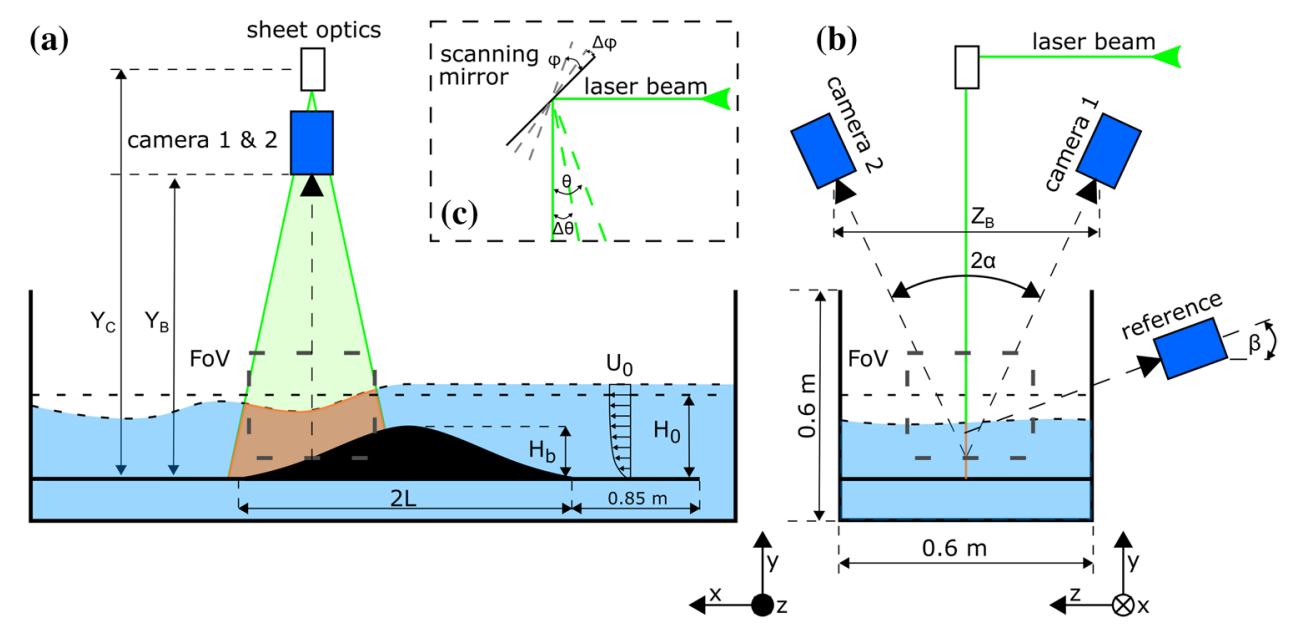

Fig. 2 Schematic of the experimental set-up. a Side-view of the setup. The wave pattern is determined by the incoming flow $\left(U_{0}\right)$, the initial water depth $\left(H_{0}\right)$, and the obstacle height $\left(H_{b}\right)$. The cameras for the PLIF and stereo-PLIF are aligned on a $y z$-plane. b Front view of the set-up, orientation of the PLIF (reference) and stereo-PLIF system (camera 1 and 2), with respect to the light sheet location. Two-

In the current work, a number of free surface wave patterns is obtained by varying the Froude number, while keeping the initial water depth constant. The free surface wave pattern is defined by the water depth above the obstacle $\left(H_{0}-H_{b}\right)$ and the upstream Froude number Fr $=U_{0} / \sqrt{g H_{0}}$, where $U_{0}$ is the upstream bulk velocity, $g$ is the gravitational acceleration, and $H_{0}$ is the initial water depth. The upstream Froude number is always sub-critical $(\mathrm{Fr}<1)$. However, transition to critical conditions $(\mathrm{Fr}=1)$ occurs at or near the obstacle (Gui et al. 2014).

The upstream flow conditions, at constant water depth $\left(H_{0}\right)$, are determined prior to the free surface wave height measurements (Table 2). The liquid velocity is measured with a disk-type programmable electromagnetic liquid velocity meter (P-EMS E30, Deltares) where one of the axes is aligned with the flow. The probe is calibrated for a velocity range of $\pm 1 \mathrm{~m} \mathrm{~s}^{-1}$ with an accuracy of $1 \%$. The initial water depth $\left(H_{0}\right)$ is measured with a ruler.

\subsection{Reference measurement}

A commonly applied PLIF system is used as a reference for the stereo-PLIF free surface measurements (Buckley and Veron 2016; Duncan et al. 1999). The light sheet from a Nd:YLF laser (LDY 304PIV laser, Litron) illuminates the liquid containing a fluorescent dye (Rhodamine WT at $120 \mathrm{mg} \mathrm{m}^{-3}$ ). The concentration of fluorescent dye is low enough such that it does not influence the static surface tension of the fluid (Appendix 1). The dynamic surface tension is more appropriate for steep waves where compression can locally alter the surfactant concentration (Duncan et al. dimensional free surface measurements are obtained with the stereoPLIF system. Reference measurements are obtained at the central light sheet location. c The light sheet is scanned with an oscillating mirror driven by a galvanometer over an angle interval of $(\Delta \theta)$ spanning a separation angle $(\theta)$

1999). However, for the current application, the static surface tension is sufficient, but the effect of compression on the surfactant concentration at a time scale similar to the wave action needs to be investigated in future work. Images are acquired with a high-speed CMOS camera (Imager HS 4M, LaVision) equipped with a $105 \mathrm{~mm}$ Micro-Nikkor objective and a high-pass filter (OG570, Schott). The magnification $\left(M_{0}\right)$ at the center plane is about $M_{0}=0.1$, with an object distance of $Z=1 \mathrm{~m}$. A large depth-of-field is obtained, which with an aperture of $f / 11$, and a wavelength of $\lambda=527 \mathrm{~nm}$, results in $\delta z \cong 4\left(1+1 / M_{0}\right)^{2} f^{\#^{2}} \lambda \approx 30 \mathrm{~mm}$ (Adrian and Westerweel 2011). The camera (Fig. 2) is placed at an angle $(\beta)$ of $15^{\circ}$ with respect to the light sheet ( $x y$-plane) to avoid interference from the liquid meniscus (Belden and Techet 2011).

An inverse, third-order polynomial is used to determine the mapping from image to world coordinates (Soloff et al. 1997). The camera is calibrated using a two-plane dot-pattern target (Type 22, LaVision). A resolution of 10.1 pixels per millimeter is obtained over a field-of-view of approximately $180 \times 180 \mathrm{~mm}^{2}$.

Table 2 Flow conditions for the free surface waves generated behind the obstacle

\begin{tabular}{lllll}
\hline Case & $U_{0} \times 10^{2}\left(\mathrm{~m} \mathrm{~s}^{-1}\right)$ & $H_{0}(\mathrm{~m})$ & $\frac{\left(H_{0}-H_{b}\right)}{H_{0}}$ & Fr $\times 10^{2}$ \\
\hline 1 & $5.23 \pm 0.17$ & 0.144 & 0.189 & $4.40 \pm 0.14$ \\
2 & $6.40 \pm 0.17$ & 0.144 & 0.189 & $5.39 \pm 0.14$ \\
3 & $11.2 \pm 0.19$ & 0.144 & 0.189 & $9.43 \pm 0.16$ \\
\hline
\end{tabular}




\subsection{Stereo-PLIF measurement}

The stereo-PLIF system extends a PLIF system to a threedimensional domain with a scanning light sheet and a stereocamera set-up. The method is comparable to conventional techniques such as scanning-PIV (Brücker 1996). Images are acquired with two high-speed CMOS cameras (Imager HS 4M, LaVision) equipped with a $105 \mathrm{~mm}$ Micro-Nikkor objective and a high-pass filter (OG570, Schott). The magnification at the center plane is approximately $M_{0}=0.1$, which with an aperture of $f / 11$ results in a depth-of-field of $\delta z \approx 30 \mathrm{~mm}$.

The cameras are placed in a stereo configuration (Fig. 2), with a full separation angle $(2 \alpha)$ of either $30^{\circ}$ or $50^{\circ}$. These two full separation angles are imposed by optical limitations in the experimental facilities, respectively, the Multiphase Wave $\mathrm{Lab}^{1}$ at MARIN in The Netherlands and the water tunnel of the Laboratory for Aero- and Hydrodynamics at the Delft University of Technology. Optimal accuracy, for conventional stereo-PIV applications, is obtained for a full separation angle of $60^{\circ} \leq 2 \alpha \leq 90^{\circ}$ (Lawson and $\mathrm{Wu} 1997$ ). The two angles are used to determine the influence of the separation angle on the measurement accuracy at comparable focal points $\left(L_{f} \approx 1 \mathrm{~m}\right)$. Consequently, the nominal magnification remains the same for both camera separation angles. Therefore, the error is expected to scale as $e_{r} \propto \sigma_{\Delta z} / \sigma_{\Delta x} \propto \tan (\alpha)^{-1}$ (Lawson and Wu 1997).

Two-dimensional free surface height measurements are obtained by scanning the light sheet in a sawtooth profile over the three-dimensional measurement domain with an oscillating mirror driven by a galvanometer (CT-6210H, Cambridge Technology) located at $y_{c}=1745 \mathrm{~mm}$ above the false bottom (Fig. 2a). The large distance of the scanning mirror ensures almost vertical measurement planes (Fig. 5a), with a maximum angle deviation, with respect to the $y$-axis, of approximately $2.3^{\circ}$ at the outer edges of the measurement domain $\left(z_{d} \approx 62 \mathrm{~mm}\right)$ (Fig. 5b).

\subsection{Edge detection procedure}

An accurate edge detection method is required for the calibration procedure and the free surface reconstruction. Large variations in image intensity $(I)$ are observed as a result of the liquid properties (Fig. 3a). The variations are a result of refraction, light focusing, and reflection at the air-water interface. Traditional edge detection can result in detection

\footnotetext{
1 The Multiphase Wave Lab is a wave flume enclosed in an autoclave, which can be operated at elevated pressure, and temperature. Furthermore, the gas surrounding the fluid can be replaced by mixtures. The optical access to the wave flume is severely limited, due to the small viewing windows of the autoclave.
}

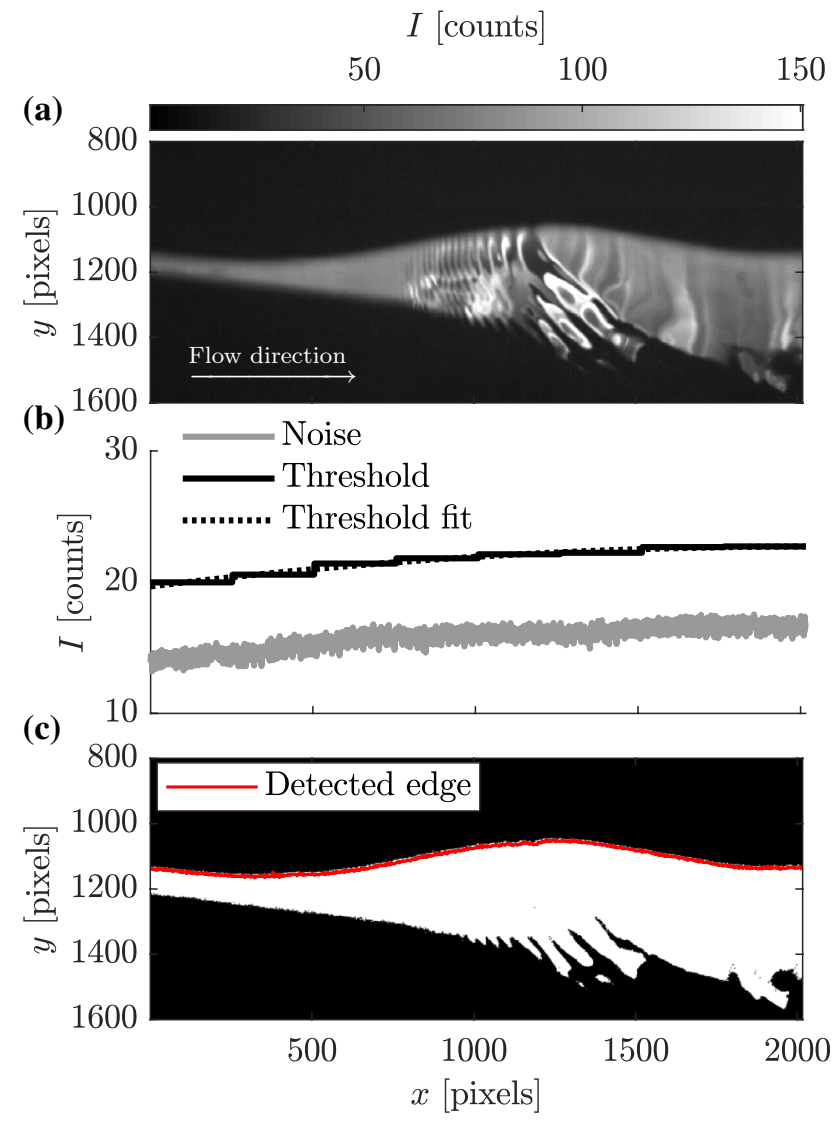

Fig. 3 Image processing steps for edge detection of the free surface waves. a The raw image $(2016 \times 2016$ pixels $)$ is cropped to show the surface waves. b The threshold distribution is determined over the top part of the image (500 pixels), which does not contain free surface information. $\mathbf{c}$ The image is thresholded and smoothed with a $(3 \times 3)$ Gaussian filter. The edge is detected based on the maximum value per column

errors due to the semi-reflective properties of the air-water interface (André and Bardet 2014).

The edge detection error depends also on the camera separation angle. The specular bias is reduced at small camera separation angles, but the quantization errors increase. Hence, there is trade-off between detection errors and quantization errors (Benetazzo 2006). Furthermore, the variation in intensity along the laser light sheet can introduce other angle dependent errors (André and Bardet 2014). These errors can be reduced with other detection methods, such as hyperbolic tangent fit methods (Hwung et al. 2009). However, the detection accuracy is not necessarily improved, and often these methods require an increased computational effort. Instead, a multi-step intensity-based detection procedure is used in the current work.

The edge information is obtained from the raw image $(2016 \times 2016$ pixels) with a multi-step intensity-based detection procedure (Fig. 3a). First, the background intensity variation, based on the windowed mean and standard deviation, 
is determined over the first part of the image (500 pixels). Then, a discontinuous threshold distribution is determined per window, which is defined as the mean plus two times the standard deviation (Fig. 3b). Finally, a continuous threshold distribution is obtained by fitting a second-order polynomial to the discontinuous data.

The raw image is binarized with the continuous threshold distribution. Then, the image is smoothed with a twodimensional $3 \times 3$ Gaussian filter to suppress the small-scale image noise (Fig. 3c). Finally, the edge is defined as the pixel with a gray level above the threshold with the lowest $y$-value (i.e. topmost "white" pixel in Fig. 3c).

Detection errors can occur with a per-column threshold operation. However, pixel data cannot be easily filtered, due to the variation in magnification over the domain. This would result in a variable filter length in world coordinates. Therefore, pixel coordinate data are processed without further filtering.

\subsection{Stereo-PLIF calibration}

The scanning stereo-PLIF system is calibrated with a selfcalibration procedure after an initial calibration at the outer edges of the measurement domain (i.e., front and aft plane). An updated mapping function is determined at each light sheet location for the two-dimensional free surface measurements.

The self-calibration procedure is similar to typical stereoPIV applications (e.g., Hori and Sakakibara 2004; Wieneke 2008). There is, however, a distinct difference. A stereoPLIF measurement contains only curvilinear lines (i.e., the intersection of the light sheet with the surface) at a specific free surface height, whereas a stereo-PIV measurement contains information over the entire field-of-view. Images of the liquid free surface are obtained at several unique still water heights, hereby sampling the entire field-of-view. The whole process of the stereo-PLIF calibration procedure is detailed in the following sections.

First, a broad calibration is performed at two planes enclosing the measurement domain (i.e., positive and negative $z$ ), shown in Fig. 4, with a two-plane dot pattern target (Type 22, LaVision). An inverse third-order polynomial mapping function $\mathbf{x}=F_{0_{i, j}}^{-1}(\mathbf{X})$ is determined for each camera (j) and plane (i), which maps pixel coordinates $\mathbf{X}=(X, Y)$ to world coordinates $\mathbf{x}=(x, y, z)$ (Soloff et al. 1997).

Then, images of the free surface are acquired at several still water heights $(N=14)$ for each light sheet location $(k)$. The edge detection procedure is applied to obtain curvilinear lines $\left(L_{k, n}\right)$ for each light sheet location $(k)$ and still water height $(n)$. These lines span the entire field-of-view.

Compared to stereo-PIV, the lines do not contain identifiable points (i.e., matching world and pixel coordinates).

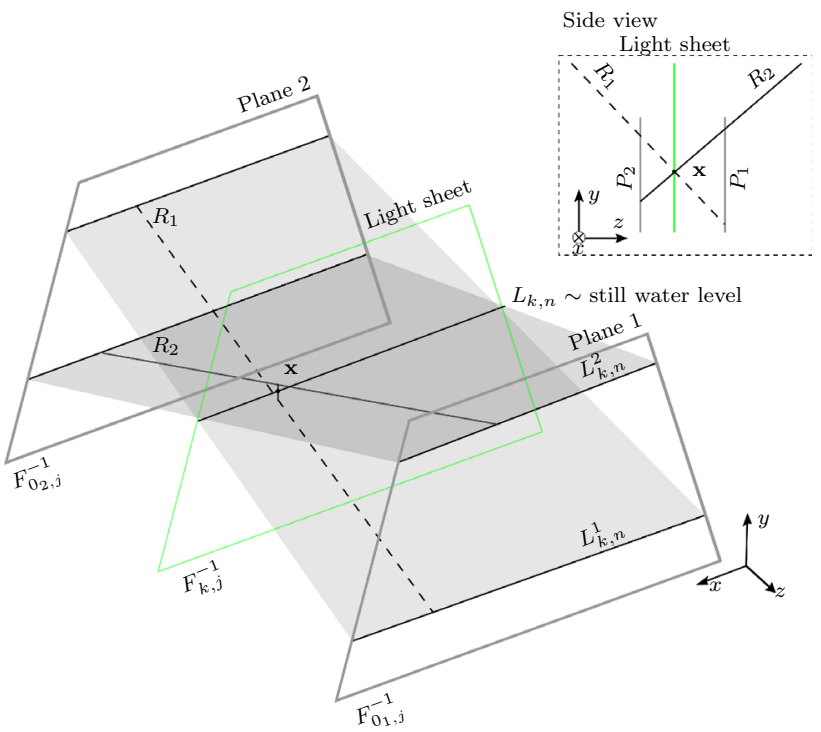

Fig. 4 Definition of variables used in the adapted self-calibration procedure. The stereo cameras are calibrated, with an initial mapping $\left(F_{0_{i j}}^{-1}\right)$, at the outer edges of the domain (i.e., Plane 1 and 2 ). The projection lines $\left(R_{j}\right)$ formed by backprojection are used to triangulate the still water level (i.e., curvilinear line $L_{k, n}$ ). An updated mapping $F_{j, k}^{-1}$ is determined at each light sheet location $(k)$ with the triangulated world $(\mathbf{x})$ and pixel $(\mathbf{X})$ coordinates. The inset shows a side view of the intersecting projection lines and the calibration planes $\left(P_{1}, P_{2}\right)$ at the outer edges of the domain

Furthermore, identifiable points can not be obtained with the approach of Hori and Sakakibara (2004), as the scanning mirror is not calibrated. Identifiable points are obtained by distance minimization between camera projection lines $\left(R_{j}(\mathbf{x})\right)$, which are the rays formed by back-projection of single pixels to the enclosing calibration planes (Fig. 4). The projection lines of both cameras are matched based on the minimal distance between skew lines with a maximum distance threshold (Gellert et al. 1989). The point of minimal distance defines the world coordinate (x) with corresponding pixel coordinates for both cameras (Fig. 4).

Next, an updated inverse polynomial mapping is determined for each camera and light sheet location $(k)$. The matched features are all constrained on a light sheet (i.e., a single plane), which enables the use of back-projection methods. The inverse mapping defines world coordinates $(x, y, 0)=F_{j, k}^{-1}(\mathbf{X})$ on a light sheet plane (Adrian and Westerweel 2011). The mapping function is completed by fitting a plane to the $z$-coordinates $(z=f(x, y))$.

Finally, the calibration domain is aligned with the liquid free surface, as the rough calibration is not necessarily aligned with the still water level. The two-dimensional free surface height $y=y(x, z)$ is determined with the calibrated stereo-PLIF system. A plane is fitted to the reconstructed 
Table 3 Resolution change over the field-of-view for the initial polynomial mapping. The initial mapping is defined at the outer edges of the domain (i.e., plane 1 and 2). The resolution change of the stereo-
PLIF system is presented per separation angle $(2 \alpha)$ and averaged over the two initial calibration planes

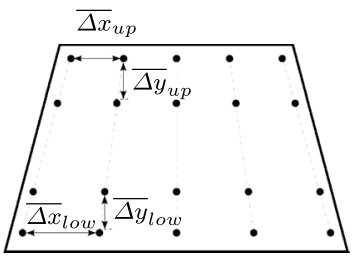

\begin{tabular}{rrrrr}
\hline $2 \alpha$ & $\overline{\Delta x}_{\text {up }}$ & $\overline{\Delta x}_{\text {low }}$ & $\overline{\Delta y}_{\text {up }}$ & $\overline{\Delta y}_{\text {low }}$ \\
& {$\left[\right.$ pixels $\mathrm{mm}^{-1}$ ] } & [pixels $\mathrm{mm}^{-1}$ ] & [pixels $\mathrm{mm}^{-1}$ ] & [pixels $\mathrm{mm}^{-1}$ ] \\
\hline 30 & 11.0 & 9.2 & 2.6 & 2.5 \\
50 & 14.2 & 11.4 & 5.4 & 5.4 \\
\hline
\end{tabular}

Fig. 5 Still water levels used for the self-calibration procedure. a Triangulated still water heights $(N=14)$ for $z_{\mathrm{d}} \approx 62 \mathrm{~mm}$, $\Delta z=3 \mathrm{~mm}$, and $2 \alpha=50^{\circ}$, showing a change in angle per light-sheet location $(k)$ and a change in domain size over the $z$-direction. b Zoom-in of the dashed box. The variation in $z$-direction is largest at the edges of the domain, with an angle of $\tan ^{-1}(3 / 75) \approx 2.3^{\circ}$ with respect to the $y$-axis (a)

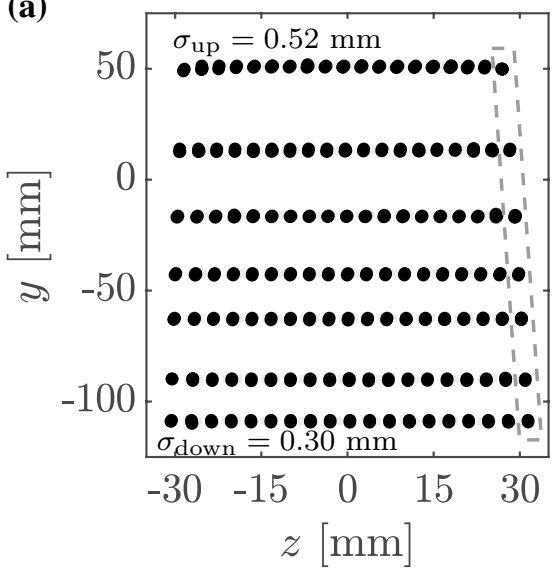

(b)

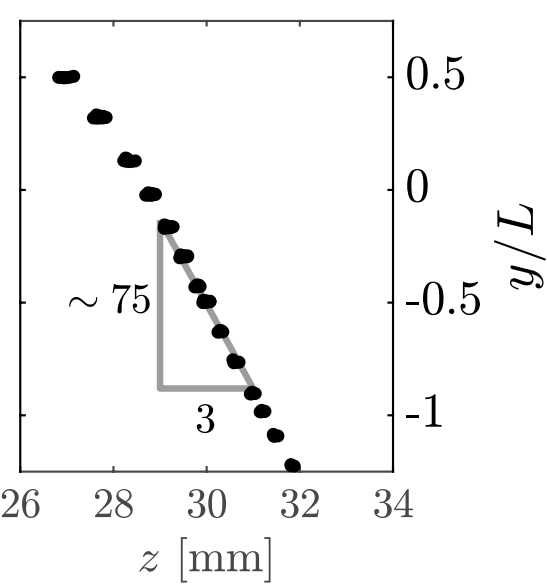

two-dimensional free surface, which is subtracted as a correction for the misalignment.

\subsection{Post-processing}

Post-processing is applied to the world coordinates obtained with both stereo cameras. The post-processing is applied to obtain uniformly distributed coordinates. Furthermore, the obtained data are filtered and averaged to obtain a combined stereo-PLIF measurement.

First, the pixel data obtained with the edge processing procedure are mapped to the respective measurement plane (i.e., light sheet location $k$ ) using the updated mapping function. The stereo camera data cannot be directly combined as the world points are non-uniformly distributed. Therefore, the world data are interpolated, with a linear interpolation method, to a uniformly distributed grid over the $x$-direction with a spacing of $\Delta x=0.1 \mathrm{~mm}$. Higher-order interpolation methods are in the current application not required, as a grid point is displaced by only $\left\|\mathbf{x}-\mathbf{x}_{i}\right\| \approx 0.04 \mathrm{~mm}$ on average.

Then, the now uniformly distributed stereo data is filtered. First, a Hampel filter, with a filter length of $L_{h, f}=2.7 \mathrm{~mm}$, is applied, which removes values that deviate more than three standard deviations from the median over the filter length (Liu et al. 2004). Then, to smooth the data, a second-order Savitzky-Golay finite impulse response filter is applied, with a filter length of $L_{h, s}=2.7 \mathrm{~mm}$ (Orfanidis 1995). Finally, the stereo-PLIF data are obtained by averaging the data from both stereo cameras.

\subsection{Calibration accuracy}

The resolution of the initial polynomial mapping is not constant over the field-of-view (Table 3). The large angle of the stereo cameras, with respect to the $z$-axis, results in a magnification change over the field-of-view. Hence, there is a significant difference between the vertical and horizontal resolutions, where the horizontal resolution changes approximately $20 \%$ over the field-of-view. Furthermore, the accuracy changes as function of the camera separation angle (Lawson and Wu 1997).

A measure of accuracy for the self-calibration procedure is the standard deviation per still water height. Therefore, a selection of still water levels used in the self-calibration procedure for a calibration domain of $z_{\mathrm{d}} \approx 62 \mathrm{~mm}$, measurement plane spacing of $\Delta z \approx 3 \mathrm{~mm}$, an initial domain size of $\Delta z_{\mathrm{d}}=80 \mathrm{~mm}$, and camera separation angle of $2 \alpha=50^{\circ}$ is shown in Fig. 5a. The standard deviation varies from $\sigma_{\text {up }}=0.52 \mathrm{~mm}$ to $\sigma_{\text {down }}=0.30 \mathrm{~mm}$ over the height $-110 \leq y \leq 50 \mathrm{~mm}$ with an average of $\bar{\sigma}=0.38 \mathrm{~mm}$; see Table 3. The higher standard deviation of $\sigma_{\text {up }}$ is either caused by the increased magnification at the top of the domain or 


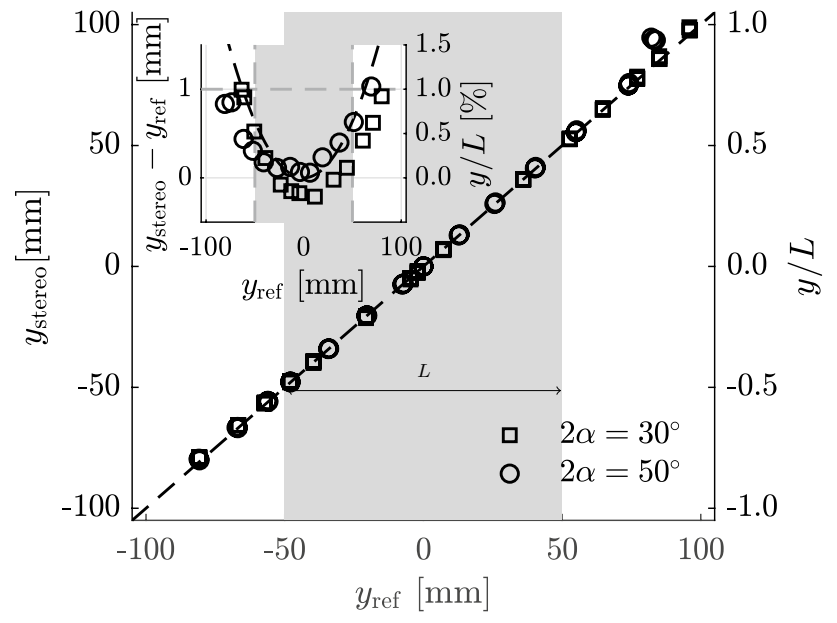

Fig. 6 The still water heights used for the self-calibration procedure are shown, per measurement domain $\left(z_{\mathrm{d}}\right)$, over the domain height (y) with the reference measurement $\left(y_{\text {ref }}\right)$ on the horizontal, and the stereo measurement $\left(y_{\text {stereo }}\right)$ on the vertical axis. To guide the eye, a dashed line is introduced as $y_{\text {stereo }}=y_{\text {ref. }}$. The inset shows the difference between $y_{\text {stereo }}-y_{\text {ref }}$ for separation angle $2 \alpha=30^{\circ}$ as open squares, and $2 \alpha=50^{\circ}$ as open circles. The $y_{\text {stereo }}$ data is normalized with the desired domain size $L$ of $100 \mathrm{~mm}$

the limited depth-of-field (i.e., the edge of the focal domain), which is evident from the larger spread over the $x$-direction (Fig. 5b). The measurement planes are positioned at a small angle, which remains minimal $\left(2.3^{\circ}\right)$ even at the outer edges of the domain (Fig. 5b). Furthermore, the angle could be removed easily by interpolating the data to a vertical plane. The virtual location of the scanning mirror, that is the intersection point of the measurement planes, is located at $y \approx 1743 \mathrm{~mm}$ above the false bottom, which corresponds with the measured value of $y_{c} \approx 1745 \mathrm{~mm}$.

The accuracy of the self-calibration procedure is further evaluated by comparing the still water levels reconstructed with the reference and stereo measurement system (Fig. 6). Measurements are performed with the stereo cameras at two different separation angles $(2 \alpha)$, while maintaining a constant focal point. The zero level correction is performed at equal still water level for both the stereo and reference measurement. However, the zero level is not equal for the two separation angles.

The free surface height is reconstructed accurately over the domain size of $-100 \leq y \leq 100 \mathrm{~mm}$ without significant influence of the domain size $\left(z_{\mathrm{d}}\right)$ and inter-plane spacing $(\Delta z)$ for both camera separation angles (Fig. 6). The difference between $y_{\text {stereo }}-y_{\text {ref }}$ over the domain size, for either separation angle $2 \alpha=30^{\circ}$ (indicated by open squares) or $2 \alpha=50^{\circ}$ (indicated by open circles), is relatively small, as shown in the inset of Fig. 6. For both separation angles, the error remains within $1 \%$ of the target domain size $(L)$ of $100 \mathrm{~mm}$. A systematic error is observed, which clearly displays a parabolic behavior.

\section{Results and discussion}

\subsection{Hydraulic flow}

The free surface waves behind an obstacle are measured at two separation angles $(2 \alpha)$ for three different Froude numbers (Table 2). The free surface wave patterns vary over a significant height, which is ideal for a proof of principle measurement. Furthermore, the wave patterns display a variety of length scales from capillary waves to large free surface undulations as shown in Fig. 7. These free surface undulations are clearly observed to travel downstream as shown in the animation (Online Resource 2).

An instantaneous snapshot of the free surface height $(y)$ is shown in Fig. 8a, d, g for the three different Froude numbers. The measurements are obtained for a domain size of $z_{\mathrm{d}} \approx 62 \mathrm{~mm}$, plane spacing of $\Delta z=3 \mathrm{~mm}$, and a separation angle of $2 \alpha=50^{\circ}$. The free surface height is reconstructed over $N_{\mathrm{p}}=19$ measurement planes with an acquisition frequency of $f_{\mathrm{a}}=f / N_{\mathrm{p}} \approx 53 \mathrm{~Hz}$ per scan, where the cameras and scanning mirror operate at $f=1 \mathrm{kHz}$.

The differences in amplitude and wavelength of the free surface waves are apparent. The temporal variance of $(\eta(x, z, t)=y-\bar{y})$, with respect to the temporal mean $\bar{y}$, is clearly observed in a space-time diagram. These space-time diagrams are obtained at two locations, spanning the $z$-direction, as shown in Fig. 8b-f, h, i).

Limited free surface disturbances, without a repetitive pattern, are expected at $x=-40 \mathrm{~mm}$ (Fig. 8b, e, h), as the upstream flow is relatively uniform. There is indeed no apparent pattern visible over the $z$-direction. However, the temporal fluctuations $(y-\bar{y})$ for the first two cases are still significant $\left(\sigma_{1}=0.34\right.$ and $\left.\sigma_{2}=0.48 \mathrm{~mm}\right)$. These periodic wave shape variations over time (Fig. 10b, e) are introduced by fluctuations in the upstream flow. For $\mathrm{Fr}=0.094$ (Fig. 10h) the velocity fluctuations have a negligible influence on the drowned hydraulic jump. Therefore, the standard deviation for the measurement at $\mathrm{Fr}=0.094$ is considered as a representable error measure, which has a magnitude of $\sigma_{3} \approx 0.15 \mathrm{~mm}$. The bias error, between the stereo and reference measurement, defined as the difference between the temporal average of the stereo and reference measurement, is $1.0 \mathrm{~mm}$ for $\mathrm{Fr}=0.094$, or $1 \%$ of the target domain size.

The second location is defined for each Froude number, to capture the changes in the free surface behavior. For Fr $=0.044$ at $x=20 \mathrm{~mm}$ (Fig. 8c), small variations in spanwise wave behavior are observed. For the $\mathrm{Fr}=0.054$ at $x=10 \mathrm{~mm}$ (Fig. 8f), the spanwise variations are more pronounced with an increased disturbance amplitude. Furthermore, large, spanwise moving, surface disturbances are observed. For the final measurement at $\mathrm{Fr}=0.094$, a 
(a)

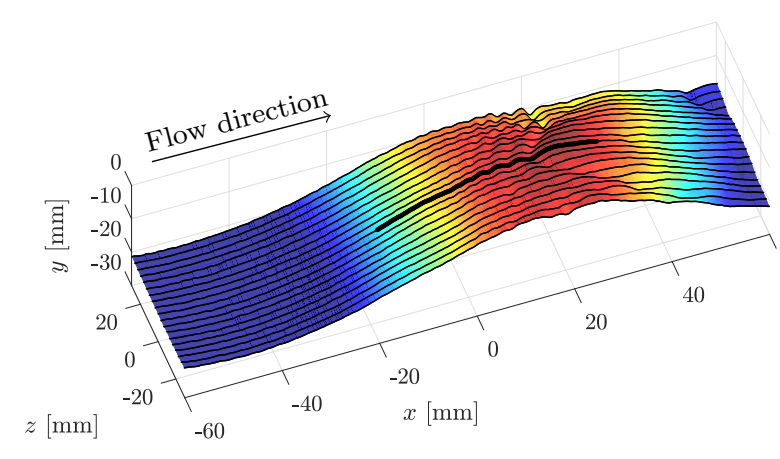

Fig. 7 a Instantaneous free surface height for $\mathrm{Fr}=0.054$. The obstacle center is located at $x \approx-80 \mathrm{~mm}$. The free surface displays typical crescent shaped disturbances, which can be clearly observed in the animation (Online Resource 2). The thick black line represents the data shown in $\mathbf{b}$. b The back-projected reference image is shown, with

relatively stationary free surface is expected. However, large free surface disturbances are observed. These disturbances occur due to air bubbles trapped on top of the recirculating flow of the submerged hydraulic jump (Te Chow 1959), which can be clearly observed in the animation (Online Resource 3).

The shape of the crescent-shaped disturbance, with preceding capillary waves, observed in Fig. 8d resembles the instabilities observed on spilling breakers (Duncan et al. 1999; Su et al. 1982). The surface disturbance shown by Gui et al. (2014) is, however, not observed with the current free surface measurements.

It is found that bubbles on the surface seriously deteriorate the signal quality, so that the measurement technique is not suited for flows with a lot of air bubbles. An example of such a detection error is shown in Fig. 9. The viewing lines of stereo-camera 1 cross the interface of an air bubble (Fig. 9a). This is registered as an additional interface above the true interface, due to the reflection of light in the air bubble. Camera 2 registers a shadow below the true interface, as the fluorescent light is reflected (Fig. 9b). However, the error introduced by the air bubble is limited (Fig. 9c). In the current work, a quality indicator (e.g., the vector norm of the distance between triangulated and mapped data) is not included, but more elaborate validation and/or processing can easily be implemented for particular situations (e.g., do not average if one of the cameras has "step-like" results).

A comparison between the temporal mean of the stereo and reference measurements is shown in Fig. 10a. The average difference is negligible $(0.2 \mathrm{~mm}$ or $0.2 \%$ of the target domain size) with a maximum deviation of $0.45 \mathrm{~mm}$. The error for $\mathrm{Fr}=0.044$ seems to be considerably larger. However, the standard deviation of the difference is, for both cases (i.e., $\sigma_{1}=0.45$ and $\sigma_{2}=0.28 \mathrm{~mm}$ ), comparable to (b)
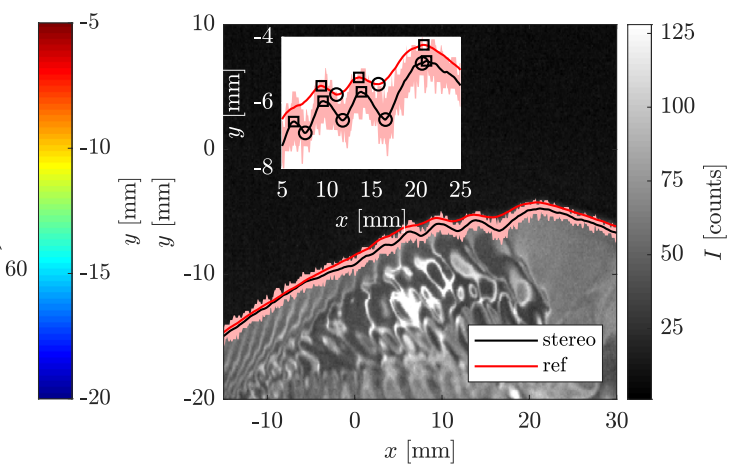

the stereo and reference data at the corresponding location. The inset shows the difference between the stereo and reference measurement, with an indication of the local peaks (open squares) and troughs (open circles). b Will be discussed later

the previous error definition. The amplitude of the free surface disturbance is estimated as $A_{1} \approx 3.2 \mathrm{~mm}$ and $A_{2} \approx 8.6 \mathrm{~mm}$, which results in a relative error $\left(\sigma_{i} / A_{i}\right)$, as defined in Table 1 , of $14 \%$ and $10 \%$ respectively. However, the relative error compared to the target domain size $\left(\sigma_{i} / L\right)$ is negligible $(\sim 0.4 \%)$. For the smaller camera separation angle $\left(2 \alpha=30^{\circ}\right)$, the averaged difference remains the same; whereas the maximum increases to $0.81 \mathrm{~mm}$. The reference camera system also has an inherent error, which for a typical PLIF system is approximately $0.5 \mathrm{~mm}$ (Duncan et al. 1999). In this case, the typical error of the reference system is estimated as the error induced by a single pixel shift, which corresponds to $0.1 \mathrm{~mm}$ or approximately $1-3 \%$ of the amplitude. The errors of the stereo-camera system are comparable to typical PLIF systems (e.g., Buckley and Veron 2017; Duncan et al. 1999).

The space-time diagrams of the disturbances $(\eta(x, 0, t)=y-\bar{y})$ are, for $\mathrm{Fr}=0.044$ and $\mathrm{Fr}=0.054$, shown in Fig. 10b, c. These results are obtained at the center line of the domain. The growth and decay of the free surface disturbances are visible. First of all, the amplitude of the free surface disturbances is observed to be larger for $\mathrm{Fr}=0.044$, whereas the occurrence frequency is higher for case one. Second, for $\mathrm{Fr}=0.054$ a crescent type of disturbance is observed, where the small amplitude waves are observed to travel upstream (towards negative $x$ ).

The local phase speed of the disturbances is, for both cases, estimated with a line as $c_{p, 1} \approx 24.7$ and $c_{p, 2} \approx 29.7 \mathrm{~cm} \mathrm{~s}^{-1}$, respectively, (Fig. 10b, c). The estimate is checked by comparing the wavelength of the disturbances on the wave crest for Fr $=0.054$ (Fig. 7a) with linear theory. A zoom-in of the disturbance is shown in Fig. 7b, where peaks (open squares) and troughs (open circles) are indicated. The wavelength $\lambda$ of the first disturbance, defined as 

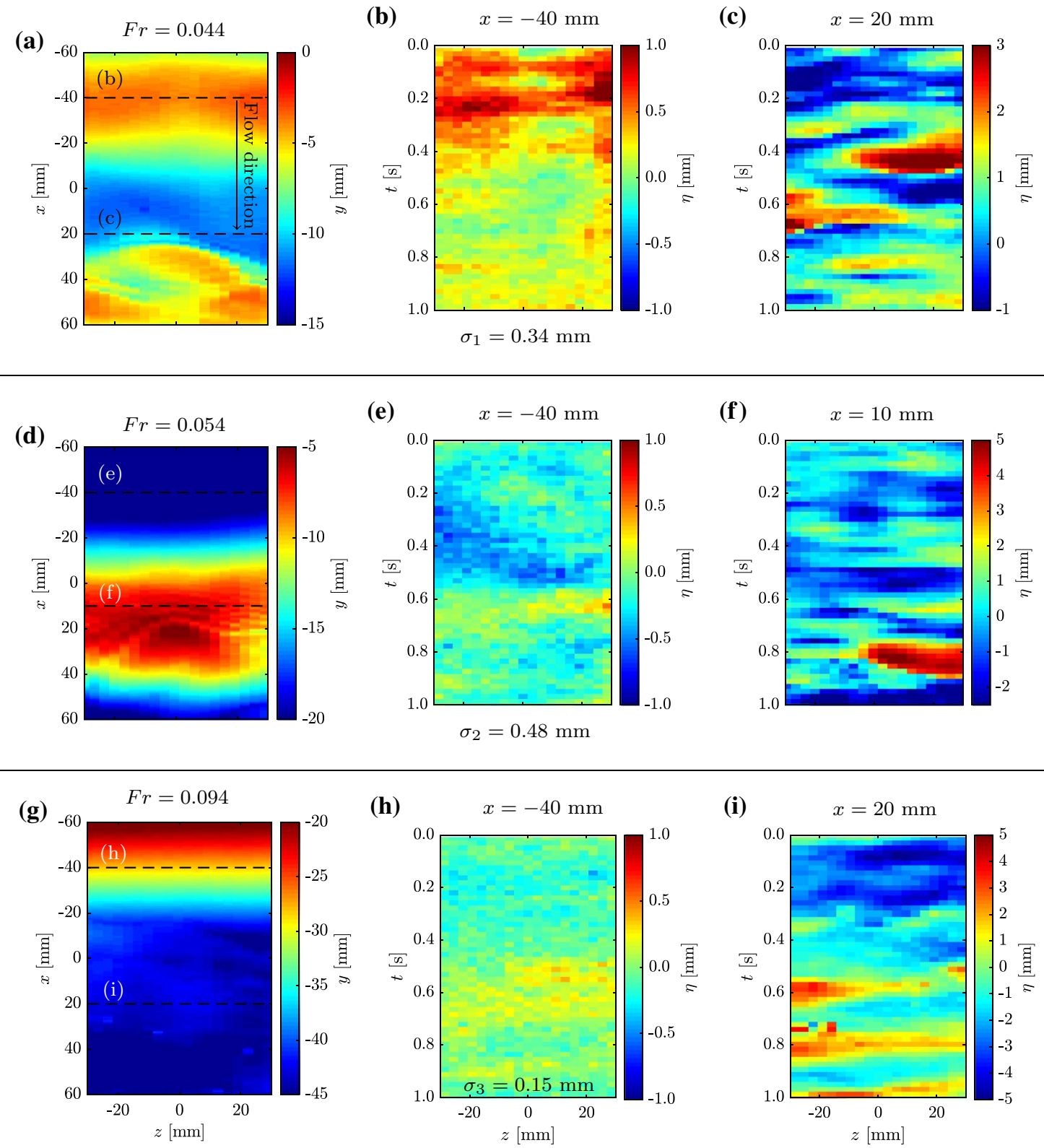

Fig. 8 Instantaneous free surface height, shown in $\mathbf{a}, \mathbf{d}, \mathbf{g}$, for different Froude numbers (Table 2). Fluid flows from negative to positive $x$ and the obstacle center is located at $x \approx-80 \mathrm{~mm}$. The space-time

the difference between crests and troughs, is approximately $\lambda_{1}=4.8 \mathrm{~mm}\left(k=1.3 \times 10^{3} \mathrm{~m}^{-1}\right)$. The dispersion relation for gravity-capillary waves on still water is,

$\omega^{2}=\left(g k+\frac{\gamma}{\rho} k^{3}\right) \tanh (k H)$,

with $\gamma$ the surface tension, $k=2 \pi / \lambda$ the wavenumber, and $H$ the water depth. For deep-water surface waves, the depth does not influence the dispersion relation (i.e., $\tanh (k H) \cong 1$ ) (Lamb 1993; Whitham 1999). The local phase velocity, diagrams of free surface height $(\eta(x, z, t)=y-\bar{y})$ at the respective $x$-coordinates per Froude number, indicated by dashed line, are shown in $\mathbf{b}, \mathbf{c}, \mathbf{e}, \mathbf{f}, \mathbf{h}, \mathbf{i})$. Note that the color scaling is different for each case

on still water, is defined as $c=\omega / k$. Based on the wavelength of the first disturbance $\left(k=1.3 \times 10^{3} \mathrm{~m}^{-1}\right)$ with surface tension and density values of $\gamma=0.072 \mathrm{~N} \mathrm{~m}^{-1}$, $\rho=998 \mathrm{~kg} \mathrm{~m}^{-3}$, respectively, the phase velocity is estimated as $c_{p, \lambda_{1}} \approx 32 \mathrm{~cm} \mathrm{~s}^{-1}$. The wavelength of the following crest is estimated as $\lambda_{2}=7.3 \mathrm{~mm}$, which results in a phase velocity of $c_{p, \lambda_{2}} \approx 28 \mathrm{~cm} \mathrm{~s}^{-1}$. The determined phase velocities are comparable to the estimate of $c_{p, 2} \approx 29.7 \mathrm{~cm} \mathrm{~s}^{-1}$ (Fig. 10c). A small difference is expected as the space-time diagram is determined in a fixed frame of reference, whereas 


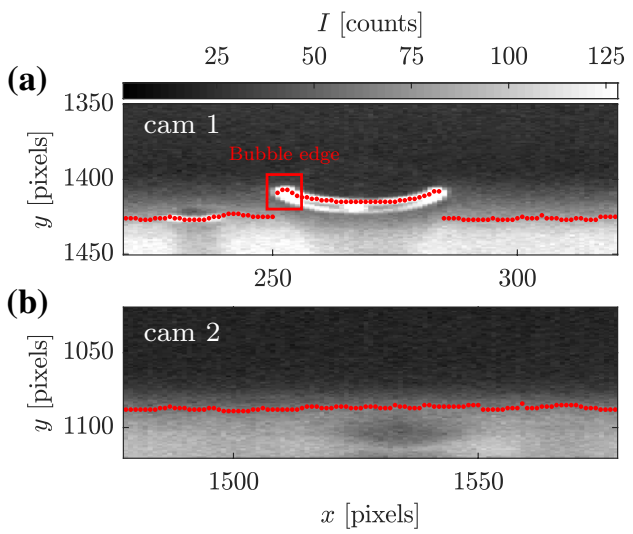

Fig. 9 Image of an edge detection error due to the presence of an air bubble on the liquid surface at the recirculation area of the drowned hydraulic jump $(\mathrm{Fr}=0.094)$. a Stereo camera 1 looks through the interface of the air bubble, which results in a detection error close to the liquid free surface. b Stereo camera 2 does not look through

the phase velocity is based on a moving frame of reference. The order of magnitude of the Doppler shift $U=c_{p, 2}-\bar{c}_{p, \lambda} \approx 2.4 \mathrm{~cm} \mathrm{~s}^{-1}$ is comparable with the upstream bulk velocity (Table 2 ). The ability of the method to measure capillary waves is indicated by the estimated wavelength and corresponding phase velocity.

\subsection{Wave swell by water drop impact}

As a second test case, a droplet impact is generated by releasing a water drop of approximately $5 \mathrm{~mm}$ in diameter from a height of $757 \mathrm{~mm}$ to impact on a deep-liquid pool $\left(H_{0}=141 \mathrm{~mm}\right)$. The resulting free surface disturbances are recorded with the stereo PLIF system in exactly the same configuration as in the first test case, with a domain size of $z_{\mathrm{d}} \approx 62 \mathrm{~mm}$, a plane spacing of $\Delta z=3 \mathrm{~mm}$, and a camera separation angle of $2 \alpha=50^{\circ}$. The measurements are timed to obtain two impact events in one recording. However, the droplet release time is not synchronized with the recording time.

The free surface disturbances resulting from the droplet impact are clearly observed in the animation (Online Resource 4). Three instantaneous snapshots of the ring waves, generated by the second impact, are shown in Fig. 11a-c. The two markers indicate the locations where time series are extracted, as shown in Fig. 11d.

The first time instance ( $t 1)$ is approximately $228 \mathrm{~ms}$ after the droplet impact at $\mathbf{X}_{p} \approx(-60.0,0.0) \mathrm{mm}$ (Fig. 11a). The maximum free surface disturbance $\eta(x, z, t)=y-\bar{y}$ is limited to $\pm 1.5 \mathrm{~mm}$ to remove the unphysical amplitude introduced by interference of either the incoming water drop or the rebounding liquid jet with the light sheet.

At the second time instance ( $t 2)$, the marker (o) is located in the trough of the first wave train (Fig. 11b). The

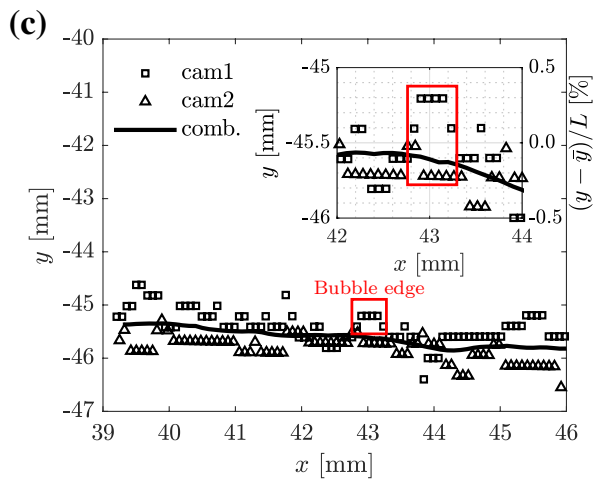

the bubble, but the bubble appears as a shadow below the interface. c The local free surface height per camera (cam 1 / 2) and filtered free surface height (comb.) are shown at the detection error location. The inset shows a zoom at the detection error location, which shows an error of approximately $0.4 \mathrm{~mm}$

small amplitude and short wavelength waves preceding the second wave train are also observed. The marker $(\triangle)$ observes the small wavelength waves preceding the first wave train. Furthermore, the second wave train generated by the droplet rebound is observed in the instantaneous snapshot of the free surface height.

Finally, the third time instance $(t 3)$ shows clearly the amplitude decay (Fig. 11c), as energy is conserved, while the front and back group velocities diverge. The amplitude decay is proportional to time as $\eta \propto t^{-1 / 2}$ (Whitham 1999).

A space-time diagram displays the free surface variation along the center line $(z=0 \mathrm{~mm})$ of the measurement domain, which is used to estimate the local phase velocity $\left(c_{\mathrm{p}}\right)$ (Fig. 12$)$. The minimum group velocity $\left(c_{g, \min }\right)$ can, however, not be estimated according to Moisy et al. (2009), as the wave amplitude is below the measurement accuracy of the current stereo-PLIF configuration.

The local phase velocities of the wave crests, resulting from the droplet impact and rebound, are estimated as $c_{\mathrm{p}} \approx 27.5 \mathrm{~cm} \mathrm{~s}^{-1}$ using the space-time diagram in Fig. 12 . The local phase velocity is larger than the minimum phase velocity for surface waves $c_{p, \min }=(4 g \gamma / \rho)^{1 / 4}=23.1 \mathrm{~cm} \mathrm{~s}^{-1}$ with a wavelength at minimum phase speed of $\lambda_{\mathrm{p}, \min }=2 \pi \sqrt{\gamma /(\rho g)}=17.3 \mathrm{~mm}$. The wavelength of the surface waves, determined as the distance between the first peak and trough at $t_{3}$ (Fig. 11c), is approximately $\lambda \approx 44 \mathrm{~mm}$. The observed waves are deep-water gravity waves $\left(\lambda<H_{0} / 2\right)$ for which the local phase velocity $c_{\mathrm{p}}=\sqrt{\left(g+\gamma \rho^{-1} k^{2}\right) / k} \approx 28.1 \mathrm{~cm} \mathrm{~s}^{-1}$ is comparable to the local phase velocity estimated from the space-time diagram shown in Fig. 12.

After, approximately, $t_{\mathrm{r}} \approx 2.6 \mathrm{~s}$ another free surface disturbance occurs (Figs. 11d, 12). This free surface disturbance is induced by the reflection of the initial wave train 


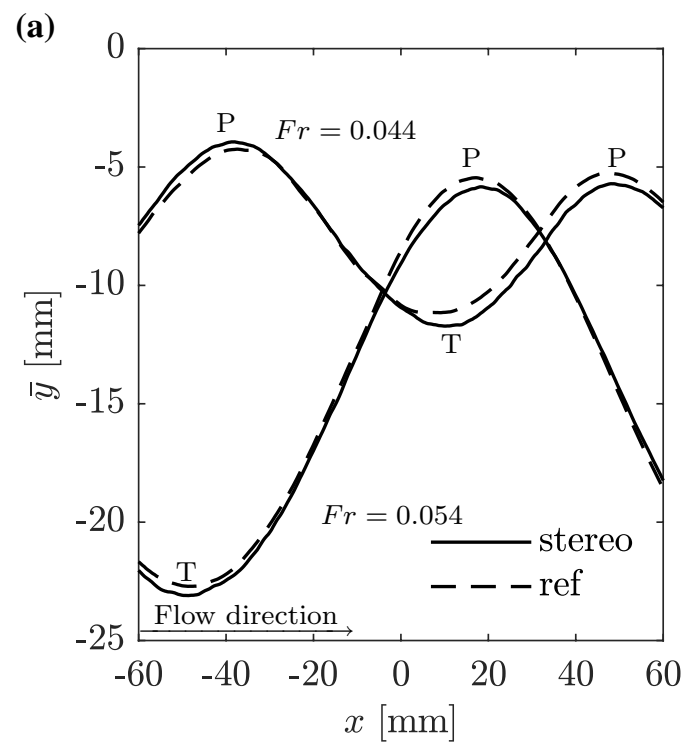

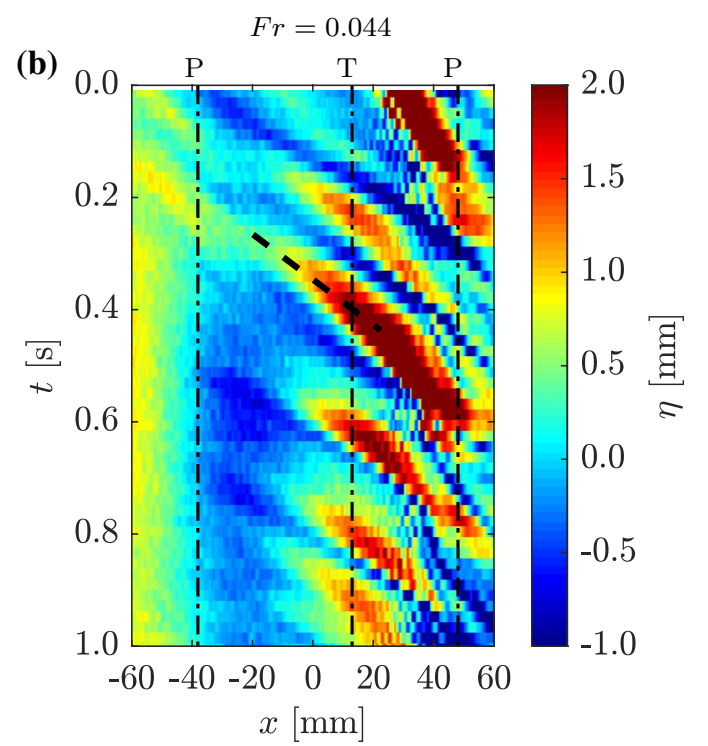

Fig. 10 a The temporal average $(\bar{y}(x, 0, t))$ of the free surface wave at $z=0$ for the stereo and reference measurement, which shows an average difference of $\overline{\bar{y}_{\text {stereo }}-\bar{y}_{\text {ref }}}=0.1 \mathrm{~mm}$, a maximum difference of $0.45 \mathrm{~mm}$, and a standard deviation of $\sigma_{1}=0.45$ and $\sigma_{2}=0.28 \mathrm{~mm}$. Space-time diagrams of the disturbance height $(\eta(x, 0, t)=y-\bar{y})$

from the side walls of the channel. The reflected wave traveled a distance of $z \approx 600 \mathrm{~mm}$ during $t_{\mathrm{r}} \approx 2.6 \mathrm{~s}$, which corresponds to a local phase velocity of $c_{\mathrm{p}} \approx 23 \mathrm{~cm} \mathrm{~s}^{-1}$. This local phase velocity is close to the minimum phase velocity $\left(c_{\mathrm{p}, \text { min }}\right)$ of surface gravity waves.

A skew is introduced in the two-dimensional free surface measurements $(\eta(x, z, t))$, as is clearly observed in Fig. 13a. The scanning system acquires line measurements over the $z$-coordinate with a temporal spacing of $\Delta t=1 \mathrm{~ms}$. In the current example, there are $N_{\mathrm{p}}=19$ light sheet locations. The difference between the first and last measurement plane is significant $(18 \mathrm{~ms})$. Based on the local

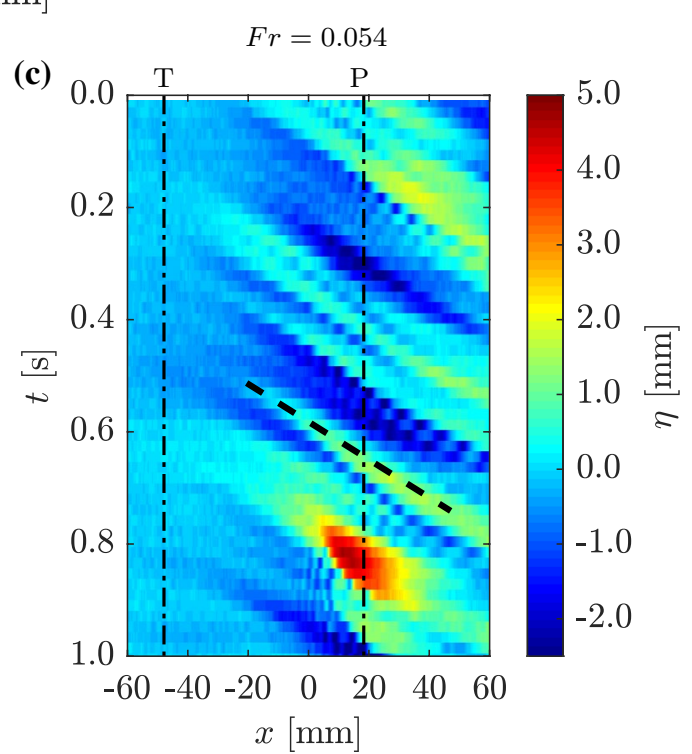

showing the growth and decay of disturbances on the free surface wave along $z=0 \mathrm{~mm}$ for $\mathbf{b} \mathrm{Fr}=0.044$ and $\mathbf{c} \mathrm{Fr}=0.054$. The location of the wave trough $(\mathrm{T})$ and peak $(\mathrm{P})$ is indicated. The thick dashed lines in $\mathbf{b}$ and $\mathbf{c}$ indicate the velocity of a disturbance wave crest $\left(c_{p, 1} \approx 24.7\right.$ and $\left.c_{p, 2} \approx 29.7 \mathrm{~cm} \mathrm{~s}^{-1}\right)$

phase velocity, this introduces a relative displacement of $\Delta x=c_{\mathrm{p}}\left(\Delta t N_{\mathrm{p}}\right) \approx 5.2 \mathrm{~mm}$. The measurements are, therefore, non-instantaneous, as the measurement time is too large which introduces a significant displacement.

Temporal interpolation can be applied to reduce the skew (Wellander et al. 2014) (Fig. 13d). The instantaneous free surface height (Fig. 13a) is interpolated to the center plane time $(t)$, where a linear change in amplitude is assumed (Fig. 13b). The absolute difference between original $(\eta)$ and interpolated $\left(\eta_{\mathrm{i}}\right)$ data shows an amplitude deviation of $\left|\eta_{\mathrm{i}}-\eta\right| \approx 0.3 \mathrm{~mm}$ with a horizontal shift of $\Delta x \approx 2.5 \mathrm{~mm}$ (Fig. 13c). The horizontal shift corresponds to the displacement based on the 
Fig. 11 Instantaneous free surface height $(\eta(x, z, t)=y-\bar{y})$, with $\bar{y}$ the temporal mean, for a droplet impact (a-c) denoted by $t_{1-3}$. The image shows spreading concentric waves. The temporal development for the points $(\circ, \triangle)$ at, respectively, $\mathbf{X}_{p}=(-20.0,0.0) \mathrm{mm}$ and $\mathbf{X}_{p}=(20.0,0.0) \mathrm{mm}$, are shown in $\mathbf{d}$. The points of $(\triangle)$ are for clarity shifted by $y=0.75 \mathrm{~mm}$. The dashed lines indicate the time instance $\left(t_{1-3}\right)$ of the instantaneous free surface heights $(\mathbf{a}-\mathbf{c})$. The dash-dotted line indicates the time $\left(t_{\mathrm{r}}\right)$ at which reflected free surface disturbances are observed

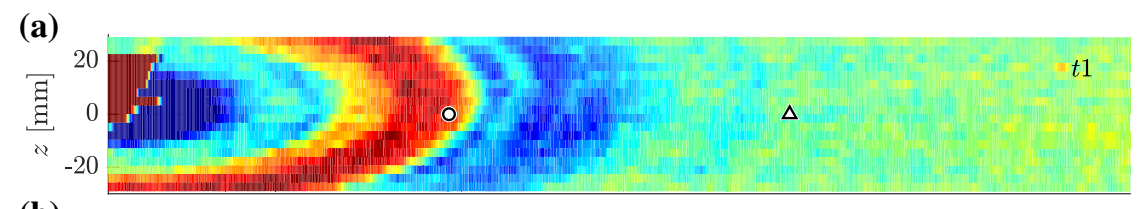

(b)

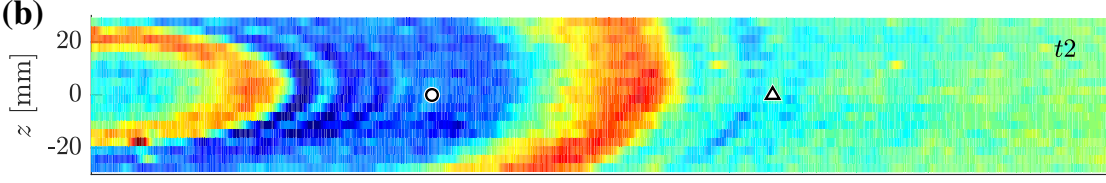

(c)

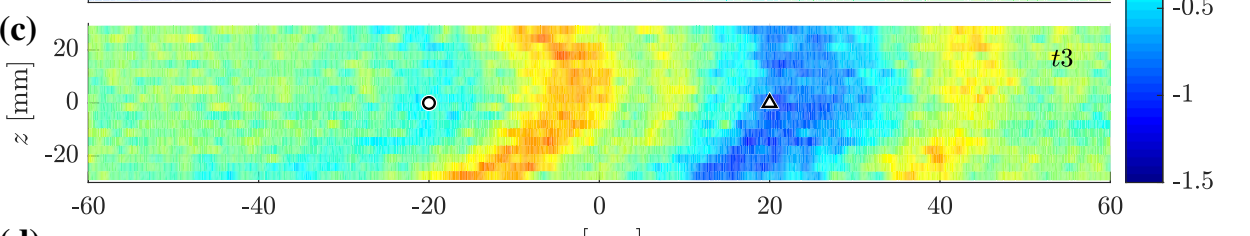

(d) $x[\mathrm{~mm}]$

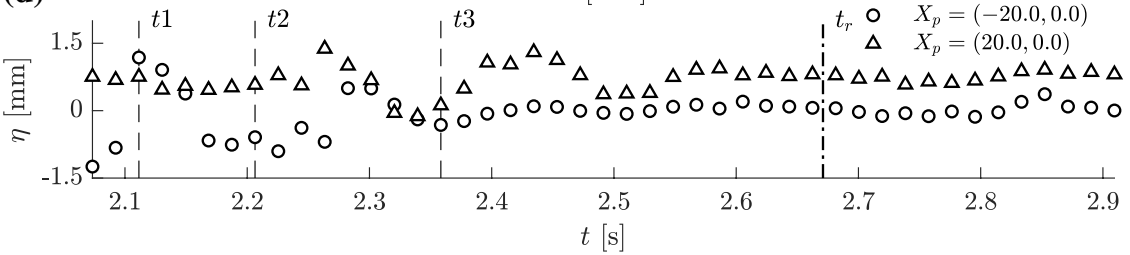
1.5 
Fig. 13 Temporal interpolation to reduce the skew introduced by scanning the three-dimensional domain. a Instantaneous free surface height $(\eta(x, z, t)=y-\bar{y})$, with $\bar{y}$ the still-water level, for a droplet impact. b Skew-corrected height $\left(\eta_{\mathrm{i}}(x, z, t)=y_{\mathrm{i}}-\bar{y}_{\mathrm{i}}\right) . \mathbf{c}$ Absolute difference between original $(\eta)$ and interpolated data $\left(\eta_{\mathrm{i}}\right)$. d Method used to interpolate data to common time $(t)$ instantaneous free surface
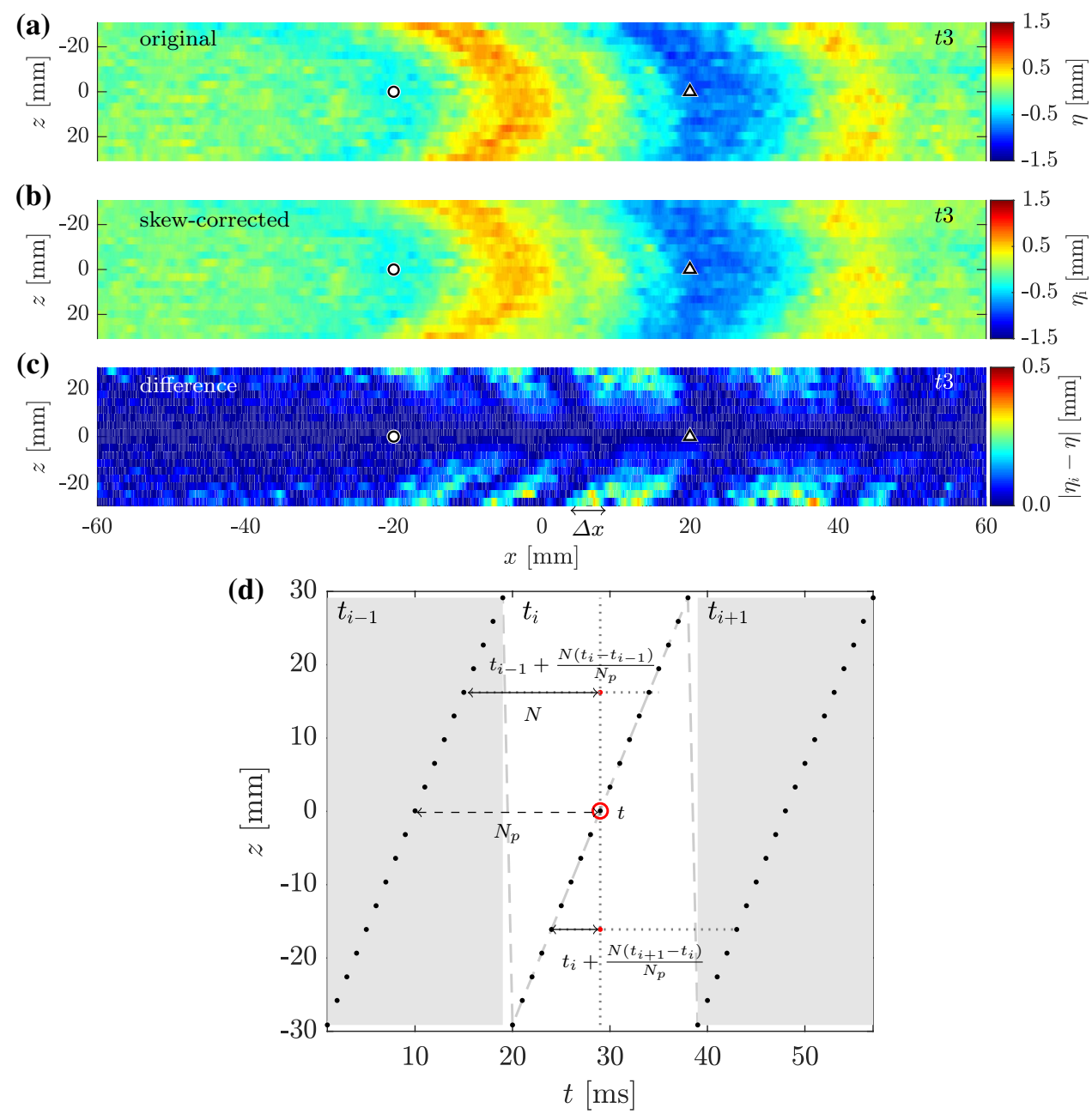

Acknowledgements This work is part of the public-private research program Sloshing of Liquefied Natural Gas (SLING) project P14-10. The support by the Netherlands Organisation for Scientific Research (NWO) Domain Applied and Engineering Sciences, and project partners is gratefully acknowledged. The authors are grateful to Mark Driessen, Nick Ebben, Victor Jaarsma, and Jan van Rijn for performing the surface tension measurements.

Open Access This article is licensed under a Creative Commons Attribution 4.0 International License, which permits use, sharing, adaptation, distribution and reproduction in any medium or format, as long as you give appropriate credit to the original author(s) and the source, provide a link to the Creative Commons licence, and indicate if changes were made. The images or other third party material in this article are included in the article's Creative Commons licence, unless indicated otherwise in a credit line to the material. If material is not included in the article's Creative Commons licence and your intended use is not permitted by statutory regulation or exceeds the permitted use, you will need to obtain permission directly from the copyright holder. To view a copy of this licence, visit http://creativecommons.org/licenses/by/4.0/.

\section{Appendix 1}

The change in fluid properties due to the addition of the particular fluorescent dye used for the stereo-PLIF is investigated. The static surface tension is measured with an adapted pendant drop method (Saad and Neumann 2016). The method minimizes the difference between the parameterized Young-Laplace equation and an experimentally obtained profile to estimate the static surface tension value. The experimental profile is determined from the shadowgraph of a pendant drop.

The pendant drop is suspended from a blunt tip needle (Fig. 14a). The droplet volume is controlled with a syringe pump (101 syringe infusion pump, KD Scientific), allowing volume adjustments of $600 \mu \mathrm{L} \mathrm{s}^{-1}$. The droplet is imaged with a CCD camera (VC-Imager Pro X 4M, LaVision) equipped with a long-distance microscope (QM 1 Long Distance Microscope, Questar). The back light illumination (OSL2 High-Intensity Fiber-Coupled Illuminator, Thorlabs) is uniform over the entire field-of-view of $5 \times 5 \mathrm{~mm}$. The 
(a)

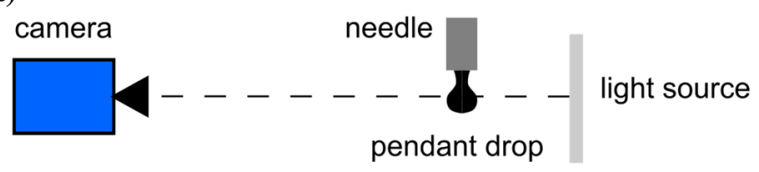

(b)

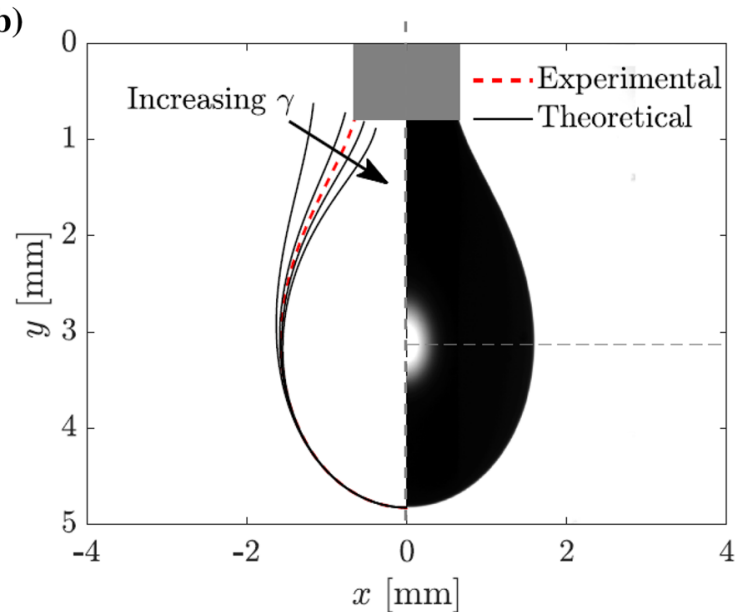

(c)

Fig. 14 Image processing to obtain the static surface tension from pendant drop images. a The experimental equipment used to obtain the shadowgraphy images. b The static surface tension of a water droplet is determined by minimizing the difference between the theoretical and experimental profile. c Image used to obtain the experimental profile for the minimization

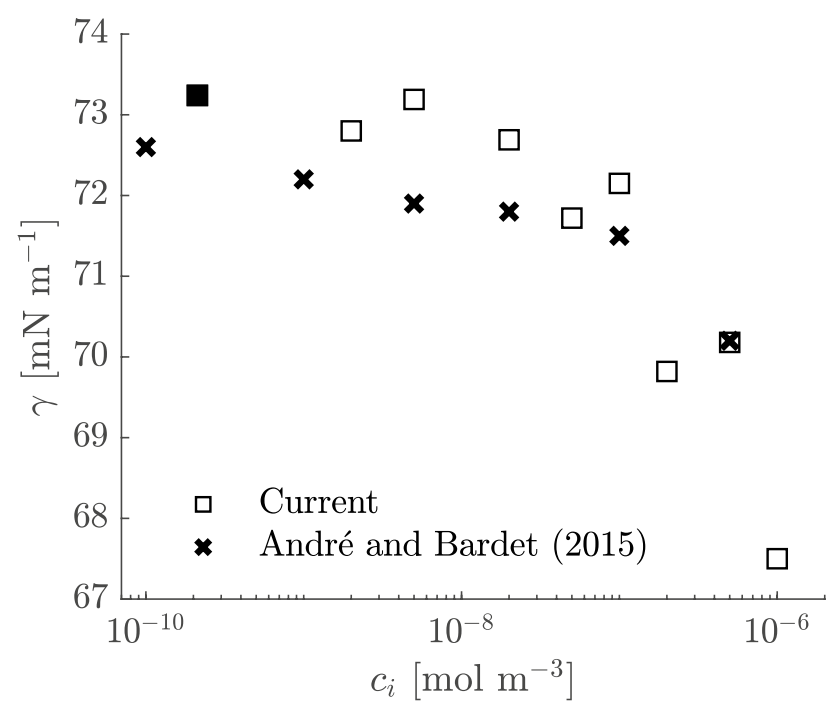

Fig. 15 Surface tension measurements with the pendant drop method. The current measurements show the dependency of surface tension on the addition of Rhodamine WT. This is compared to the dependency of Rhodamine 6G (André and Bardet 2015). The concentration used in the experiments is indicated by the filled marker ( $\bullet$ ) edge is easily obtained with a gradient-based edge detection procedure (Fig. 14c).

The static surface tension is determined by minimizing the difference between the theoretical and experimental profile (Fig. 14b). The static surface tension of water-air at $T=21.3^{\circ} \mathrm{C}$ is measured as $\gamma=72.58 \mathrm{mN} \mathrm{m}^{-1}$. The influence of Rhodamine $6 \mathrm{G}$ with increasing molar concentration $\left(c_{\mathrm{i}}\right)$ has previously been investigated, where it was shown that there is a negligible effect for practical concentrations (André and Bardet 2015). The influence of Rhodamine WT is investigated over a similar concentration span (Fig. 15). The static surface tension value is slightly overestimated, but the trend is similar to the previous work. The effect on the static surface tension is limited for the current application of the stereo-PLIF system, as indicated by the filled marker (-) (Fig. 15). However, the dynamic surface tension needs to investigated for cases where compression can locally alter the surfactant concentration.

\section{References}

Adrian RJ, Westerweel J (2011) Particle image velocimetry, vol 30. Cambridge University Press, Cambridge. https://doi. org/10.1007/978-3-319-68852-7

André MA, Bardet PM (2014) Velocity field, surface profile and curvature resolution of steep and short free-surface waves. Exp Fluids 55(4):1709. https://doi.org/10.1007/s00348-014-1709-5

André MA, Bardet PM (2015) Interfacial shear stress measurement using high spatial resolution multiphase PIV. Exp Fluids 56(6). https://doi.org/10.1007/s00348-015-2006-7

Belden J, Techet AH (2011) Simultaneous quantitative flow measurement using PIV on both sides of the air-water interface for breaking waves. Exp Fluids 50(1):149-161. https://doi.org/10.1007/ s00348-010-0901-5

Benetazzo A (2006) Measurements of short water waves using stereo matched image sequences. Coast Eng 53(12):1013-1032. https:// doi.org/10.1016/j.coastaleng.2006.06.012

Benetazzo A, Fedele F, Gallego G, Shih PC, Yezzi A (2012) Offshore stereo measurements of gravity waves. Coast Eng 64:127-138. https://doi.org/10.1016/j.coastaleng.2012.01.007

Brücker C (1996) 3-D scanning-particle-image-velocimetry: technique and application to a spherical cap wake flow. Appl Sci Res 56(23):157-179. https://doi.org/10.1007/bf02249379

Buckley MP, Veron F (2016) Structure of the airflow above surface waves. J Phys Oceanogr 46(5):1377-1397. https://doi. org/10.1175/jpo-d-15-0135.1

Buckley MP, Veron F (2017) Airflow measurements at a wavy airwater interface using PIV and LIF. Exp Fluids 58(11). https://doi. org/10.1007/s00348-017-2439-2

Cobelli PJ, Maurel A, Pagneux V, Petitjeans P (2009) Global measurement of water waves by Fourier transform profilometry. Exp Fluids 46(6):1037-1047. https://doi.org/10.1007/s00348-009-0611-z

Dabiri D, Gharib M (2001) Simultaneous free-surface deformation and near-surface velocity measurements. Exp Fluids 30(4):381-390. https://doi.org/10.1007/s003480000212

Douxchamps D, Devriendt D, Capart H, Craeye C, Macq B, Zech Y (2005) Stereoscopic and velocimetric reconstructions of the free 
surface topography of antidune flows. Exp Fluids 39(3):535-553. https://doi.org/10.1007/s00348-005-0983-7

Duncan JH, Qiao H, Philomin V, Wenz A (1999) Gentle spilling breakers: crest profile evolution. J Fluid Mech 379:191-222. https://doi. org/10.1017/s0022112098003152

Gellert W, Gottwald S, Hellwich M, Kästner H, Küstner H (eds) (1989) VNR concise encyclopedia of mathematics. Van Nostrand Reinhold

Gomit G, Chatellier L, Calluaud D, David L (2013) Free surface measurement by stereo-refraction. Exp Fluids 54(6). https://doi. org/10.1007/s00348-013-1540-4

Gomit G, Chatellier L, Calluaud D, David L, Fréchou D, Boucheron R, Perelman O, Hubert C (2015) Large-scale free surface measurement for the analysis of ship waves in a towing tank. Exp Fluids 56(10). https://doi.org/10.1007/s00348-015-2054-z

Gui L, Yoon H, Stern F (2014) Techniques for measuring bulge-scar pattern of free surface deformation and related velocity distribution in shallow water flow over a bump. Exp Fluids 55(4):1721. https://doi.org/10.1007/s00348-014-1721-9

Hori T, Sakakibara J (2004) high-speed scanning stereoscopic PIV for $3 \mathrm{~d}$ vorticity measurement in liquids. Meas Sci Technol 15(6):1067-1078. https://doi.org/10.1088/0957-0233/15/6/005

Hwung HH, Kuo CA, Chien CH (2009) Water surface level profile estimation by image analysis with varying overhead camera posture angle. Meas Sci Technol 20(7):075104. https://doi. org/10.1088/0957-0233/20/7/075104

Jähne B, Haußecker H (1998) air-water gas exchange. Annu Rev Fluid Mech 30(1):443-468. https://doi.org/10.1146/annurev.fluid .30 .1 .443

Lafeber W, Bogaert H, Brosset L (2012) comparison of wave impact tests at large and full scale: results from the Sloshel project. In: The twenty-second international offshore and polar engineering conference

Lamb H (1993) Hydrodynamics. Cambridge University Press, Cambridge

Lawson NJ, Wu J (1997) Three-dimensional particle image velocimetry: experimental error analysis of a digital angular stereoscopic system. Meas Sci Technol 8(12):1455-1464. https://doi. org/10.1088/0957-0233/8/12/009

Liu J, Paul JD, Gollub JP (1993) Measurements of the primary instabilities of film flows. J Fluid Mech 250(-1):69. https://doi. org/10.1017/S0022112093001387

Liu H, Shah S, Jiang W (2004) On-line outlier detection and data cleaning. Comput Chem Eng 28(9):1635-1647. https://doi. org/10.1016/j.compchemeng.2004.01.009
Moisy F, Rabaud M, Salsac K (2009) A synthetic Schlieren method for the measurement of the topography of a liquid interface. Exp Fluids 46(6): 1021. https://doi.org/10.1007/s00348-008-0608-z

Orfanidis S (1995) Introduction to signal processing. Prentice Hall, Englewood Cliffs

Saad SM, Neumann AW (2016) Axisymmetric drop shape analysis (ADSA): an outline. Adv Colloid Interface Sci 238:62-87. https ://doi.org/10.1016/j.cis.2016.11.001

Savelsberg R, Holten A, van de Water W (2006) Measurement of the gradient field of a turbulent free surface. Exp Fluids 41(4):629_ 640. https://doi.org/10.1007/s00348-006-0186-x

Soloff SM, Adrian RJ, Liu ZC (1997) Distortion compensation for generalized stereoscopic particle image velocimetry. Meas Sci Technol 8(12):1441. https://doi.org/10.1088/0957-0233/8/12/008

Su MY, Bergin M, Marler P, Myrick R (1982) Experiments on nonlinear instabilities and evolution of steep gravity-wave trains. J Fluid Mech 124:45-72. https://doi.org/10.1017/s0022112082002407

Te Chow V (1959) Open-channel hydraulics, vol 1. McGraw-Hill, New York

Tsubaki R, Fujita I (2005) Stereoscopic measurement of a fluctuating free surface with discontinuities. Meas Sci Technol 16(10):18941902. https://doi.org/10.1088/0957-0233/16/10/003

Turney DE, Anderer A, Banerjee S (2009) a method for three-dimensional interfacial particle image velocimetry (3d-IPIV) of an air-water interface. Meas Sci Technol 20(4):045403. https://doi. org/10.1088/0957-0233/20/4/045403

Wellander R, Richter M, Aldén M (2014) time-resolved (kHz) 3D imaging of OH PLIF in a flame. Exp Fluids 55(6). https://doi. org/10.1007/s00348-014-1764-y

Whitham GB (1999) Linear and nonlinear waves. Wiley, New York

Wieneke B (2008) Volume self-calibration for 3D particle image velocimetry. Exp Fluids 45(4):549-556. https://doi.org/10.1007/ s00348-008-0521-5

Zavadsky A, Benetazzo A, Shemer L (2017) On the two-dimensional structure of short gravity waves in a wind wave tank. Phys Fluids 29(1):016601. https://doi.org/10.1063/1.4973319

Publisher's Note Springer Nature remains neutral with regard to jurisdictional claims in published maps and institutional affiliations. 